\title{
Article \\ Incidence and Practice of Early Prone Positioning in Invasively Ventilated COVID-19 Patients-Insights from the PRoVENT-COVID Observational Study
}

\author{
Willemke Stilma ${ }^{1,2, *,+}$, , David M. P. van Meenen ${ }^{1,+}$, Christel M. A. Valk ${ }^{1}$, Hendrik de Bruin ${ }^{1}$, \\ Frederique Paulus ${ }^{1,2}$, Ary Serpa Neto ${ }^{3,4}\left(\mathbb{D}\right.$, Marcus J. Schultz ${ }^{1,5,6}$ and on behalf of the PRoVENT-COVID \\ Collaborative Group $\ddagger, \S$
}

Citation: Stilma, W.; van Meenen, D.M.P.; Valk, C.M.A.; de Bruin, H.; Paulus, F.; Serpa Neto, A.; Schultz, M.J.; on behalf of the PRoVENTCOVID Collaborative Group. Incidence and Practice of Early Prone Positioning in Invasively Ventilated COVID-19 Patients-Insights from the PRoVENT-COVID Observational Study. J. Clin. Med. 2021, 10, 4783. https://doi.org/10.3390/jcm10204783

Academic Editor: Luca Brazzi

Received: 30 July 2021

Accepted: 12 October 2021

Published: 19 October 202

Publisher's Note: MDPI stays neutral with regard to jurisdictional claims in published maps and institutional affiliations.

Copyright: (c) 2021 by the authors. Licensee MDPI, Basel, Switzerland. This article is an open access article distributed under the terms and conditions of the Creative Commons Attribution (CC BY) license (https:/ / creativecommons.org/licenses/by/ $4.0 /)$.
1 Department of Intensive Care, Amsterdam UMC, Location 'AMC', 1105 AZ Amsterdam, The Netherlands; d.m.vanmeenen@amsterdamumc.nl (D.M.P.v.M.); c.m.valk@amsterdamumc.nl (C.M.A.V.); h.debruin2@amsterdamumc.nl (H.d.B.); f.paulus@amsterdamumc.nl (F.P.); marcus.j.schultz@gmail.com (M.J.S.)

2 Center of Expertise Urban Vitality, Faculty of Health, Amsterdam University of Applied Sciences, 1105 BD Amsterdam, The Netherlands

3 Australian and New Zealand Intensive Care Research Centre (ANZIC-RC), Monash University, Melbourne 3004, Australia; ary.serpaneto@monash.edu

4 Department of Critical Care Medicine, Hospital Israelita Albert Einstein, São Paulo 05652-900, Brazil

5 Mahidol-Oxford Research Unit (MORU), Faculty of Tropical Medicine, Mahidol University, Bangkok 10400, Thailand

6 Nuffield Department of Medicine, University of Oxford, Oxford OX3 7BN, UK

* Correspondence: w.stilma@amsterdamumc.nl

+ These authors contributed equally to this work.

$\ddagger$ PRactice of VENTilation in COVID-19 study.

$\S$ Collaborative authors are presented in Appendix A.

\begin{abstract}
We describe the incidence and practice of prone positioning and determined the association of use of prone positioning with outcomes in invasively ventilated patients with acute respiratory distress syndrome (ARDS) due to coronavirus disease 2019 (COVID-19) in a national, multicenter observational study, performed at 22 intensive care units in the Netherlands. Patients were categorized into 4 groups, based on indication for and actual use of prone positioning. The primary outcome was 28-day mortality. Secondary endpoints were 90-day mortality, and ICU and hospital length of stay. In 734 patients, prone positioning was indicated in $60 \%$-the incidence of prone positioning was higher in patients with an indication than in patients without an indication for prone positioning (77 vs. $48 \%, p=0.001$ ). Patients were left in the prone position for median 15.0 (10.5-21.0) hours per full calendar day-the duration was longer in patients with an indication than in patients without an indication for prone positioning (16.0 (11.0-23.0) vs. 14.0 (10.0-19.0) hours, $p<0.001)$. Ventilator settings and ventilation parameters were not different between the four groups, except for $\mathrm{FiO}_{2}$ which was higher in patients having an indication for and actually receiving prone positioning. Our data showed no difference in mortality at day 28 between the 4 groups (HR no indication, no prone vs. no indication, prone vs. indication, no prone vs. indication, prone: 1.05 (0.76-1.45) vs. 0.88 $(0.62-1.26)$ vs. $1.15(0.80-1.54)$ vs. $0.96(0.73-1.26)(p=0.08))$. Factors associated with the use of prone positioning were ARDS severity and $\mathrm{FiO}_{2}$. The findings of this study are that prone positioning is often used in COVID-19 patients, even in patients that have no indication for this intervention. Sessions of prone positioning lasted long. Use of prone positioning may affect outcomes.
\end{abstract}

Keywords: coronavirus disease 2019; COVID-19; ARDS; prone positioning; intensive care; critical care; artificial ventilation; mortality 


\section{Introduction}

Patients with acute respiratory distress syndrome (ARDS) have been shown to benefit from early prone positioning if hypoxemia is severe and refractory through an increase in the fraction of inspired oxygen $\left(\mathrm{FiO}_{2}\right)>60 \%$ and higher positive end-expiratory pressure (PEEP) [1,2]. Especially patients with focal consolidations could profit from this intervention [3], as higher PEEP may be ineffective and could even cause overdistension. Before the coronavirus disease 2019 (COVID-19) pandemic, prone positioning remained remarkably underused $[4,5]$.

Invasively ventilated patients with ARDS due to COVID-19 often have an indication for prone positioning. Indeed, these patients often have severe hypoxemia. Additionally, consolidation may behave as focal lesions [6,7], which is another reason to apply prone positioning early after the start of invasive ventilation [8]. Last but not least, hypoxemia could also be a consequence of pulmonary embolism, for which higher PEEP is not helpful. Several recent reports in COVID-19 patients have shown frequent use of prone positioning, but with a remarkable variance in incidence and practice [6,8-11].

The purpose of this current analysis of a national multicenter study of COVID-19 patients admitted to the intensive care unit (ICU) for invasive ventilation early in the pandemic, named 'PRactice of VENTilation in COVID-19' (PRoVENT-COVID) [12], was to study the incidence and practice of prone positioning in this cohort. We tested the hypothesis that prone positioning improves the outcome of COVID-19 patients. We also wished to determine what factors were associated with its use.

\section{Methods}

\subsection{Study Design}

The PRoVENT-COVID study is an observational cohort study undertaken at 22 ICUs from 1 March 2020 until 1 June 2020 in the Netherlands-in this study, we enrolled 40\% of all patients that needed invasive ventilation during the first wave of the national outbreak [13]. The study protocol [12] and the statistical analysis plan for the current analysis were prepublished [14].

\subsection{Ethics}

The study protocol was approved by the ethics committee in Amsterdam UMC, Amsterdam, the Netherlands (registration number W20_157 \# 20.171); the need for individual patient informed consent was waived due to the observational nature of the study.

\subsection{Study Registration}

The study was registered at clinicaltrials.gov on 15 April 2020 with study identifier NCT04346342.

\subsection{Inclusion and Exclusion Criteria}

Consecutive patients were enrolled in the PRoVENT-COVID study if (1) age was $>18$ years; (2) they were admitted to one of the participating ICUs; and (3) they had received invasive ventilation for respiratory failure related to COVID-19 that was confirmed by a reverse transcriptase-polymerase chain reaction for SARS-CoV-2. The PRoVENT-COVID study had no exclusion criteria. For the current analysis, we excluded patients who were transferred from or to another ICU during the first days of invasive ventilation, as it could be that prone positioning was delayed because of an imminent transport and also because data on the use of prone positioning could not be assessed in non-participating centers.

\subsection{Collected Data, and Patient Classification}

We collected demographic data, including disease severities and the medical history at baseline. ARDS severity was scored as mild, moderate or severe, in accordance with the current definition for ARDS [15]. Ventilator settings and ventilation parameters were collected every $8 \mathrm{~h}$, and use and timing of prone positioning and use of neuromuscular 
blocking agents (NMBA) were collected in the first 4 calendar days of invasive ventilation. Chest $\mathrm{X}$-rays and lung CT-scans were made at initiation of invasive ventilation. The $\mathrm{X}$-rays were coded in quadrants and the CT-scans as a \%. This was based on the interpretation of trained data collectors: all data collectors had a medical background and had received additional training regarding the chest X-rays and lung CT-scan assessment before the start of data collection. Follow-up was complete up to day 90 , and included timing of liberation from invasive ventilation, ICU and hospital discharge, and life status at ICU and hospital discharge, and at day 28 and day 90.

Patients were categorized into 4 groups based on indication for (yes or no) and the use of (yes or no) early prone positioning. A patient was labeled to have an indication for prone positioning if $\mathrm{PaO}_{2} / \mathrm{FiO}_{2}$ ratio $<150 \mathrm{mmHg}$, at PEEP of $\geq 5 \mathrm{~cm} \mathrm{H}_{2} \mathrm{O}$ and $\mathrm{FiO}_{2} \geq 0.6$ [1] for at least 2 consecutive time points within the first $32 \mathrm{~h}$ after the start of invasive ventilation.

\subsection{Study Endpoints}

The primary endpoint of this analysis was 28-day mortality. Secondary outcomes were 90-day mortality, and ICU- and hospital length of stay (LOS).

\subsection{Statistical Analyses}

We did not perform a formal sample size calculation; instead, the number of available patients served as the sample size. The day of intubation, which in theory could last from $1 \mathrm{~min}$ to $23 \mathrm{~h}$ and $59 \mathrm{~min}$, was named 'day 0 '. Successive days were named 'day 1 ', 'day 2' and 'day 3'.

Categorical patient variables are presented as numbers and percentages, and continuous data as medians with interquartile ranges. With regard to the primary endpoint, there were no missing data. The amount of missing data of other variables was low, $<5 \%$. Incidence of prone positioning is expressed as numbers and percentages variables. Timing and duration of prone positioning are expressed in the number of hours from the start of invasive ventilation, and the total number of hours per full calendar day. To assess differences among the 4 groups a chi-squared test and Kruskal-Wallis test were used where appropriate.

Ventilatory variables and parameters over the first 4 calendar days were compared using a Kruskal-Wallis test and were presented in cumulative distribution plots and line graphs displaying the 4 groups of interest. For each day, ventilatory variables at the moment of the worst $\mathrm{PaO}_{2} / \mathrm{FiO}_{2}$ for that day were used, assuming these were collected at the moment the patient was in a supine position.

Hazard ratios (HRs) for 28-day and 90-day mortality were compared between the 4 groups using a (shared-frailty) Cox proportional hazard model, with the center as frailty. HRs for ICU length of stay, hospital length of stay and ventilator free days, were compared using a competing risk analysis with the center as a random effect. Kaplan-Meier curves were constructed for all outcomes of interest. Predefined variables assessed for the final models were severity of ARDS [10], $\mathrm{PEEP} \mathrm{FiO}_{2}$, body mass index (BMI), use of NMBAs and tidal volume per predicted body weight. If these variables had a $p<0.20$ in the univariable model, they were included in the multivariable model. Covariates used for the final model were the variables with a $p<0.05$ in the multivariable model; the covariates used in the univariable, and multivariable models are reported in Table S1. This analysis was repeated to compare patients having an indication for and receiving prone positioning and patients having an indication for but not receiving prone positioning.

An adjusted mixed-effect model with the center as a random effect was used to determine which factors had an association with use of prone positioning. Variables included in this model were severity of ARDS [16], PEEP, $\mathrm{FiO}_{2}$, body mass index (BMI) and hypercapnia.

As a posthoc analysis, a time-dependent Cox regression analysis was performed. All models were checked for collinearity. All analyses were conducted in R v.4.0.3 ( $\mathrm{R}$ 
Foundation for Statistical Computing: Vienna, Austria) [17] and a $p<0.05$ was considered significant.

\section{Results}

\subsection{Patients Enrolled}

Between 1 March and 1 June 2020, 22 ICUs were invited and accepted participation in the PRoVENT-COVID study. Of 1122 enrolled patients, 734 patients were eligible for the current analysis. The main reason for exclusion was an early transfer from or to a non-participating hospital (Figure 1). At the start of ventilation, patients that were placed in prone positioning had higher severities of ARDS, and $\mathrm{PaO}_{2} / \mathrm{FiO}_{2}<150$ was more frequent in patients that had an indication for prone positioning (Table 1). Additionally, in the group without an indication for prone positioning, the severity of ARDS and the number of patients with a $\mathrm{PaO}_{2} / \mathrm{FiO}_{2} \leq 150 \mathrm{~mm} \mathrm{Hg}$ was higher in the group that received prone positioning than the group that was not placed in the prone position (Table 1). NMBAs were used more often in patients having an indication than in patients not having an indication for prone positioning ( $60 \%$ vs. $52 \%)$.

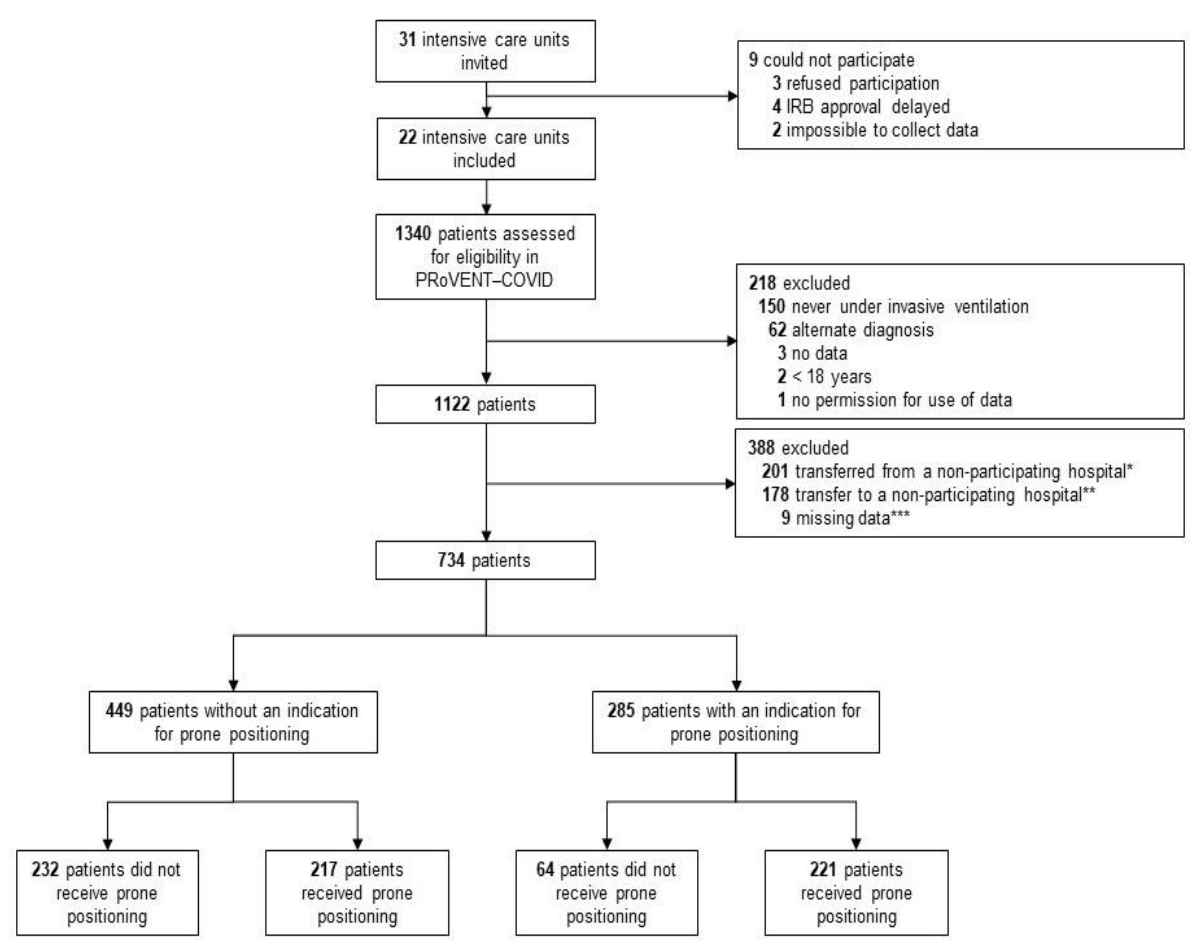

Figure 1. Flow chart of patient inclusion.

\subsection{Incidence of Prone Positioning}

Prone positioning was used in 438/734 (60\%) patients. Incidence of prone positioning was higher in patients having an indication than in patients not having an indication for prone positioning $(77 \%$ vs. $48 \% ; p<0.001)$. For patients who were placed in the prone position, the median first day of proning was day $0(0-1)$ and received prone positioning over a median of 3 (2-3) days; this was similar in patients with and without an indication. Prone positioning lasted a median of $16.0(11.0-23.0)$ hours per full calendar day in patients having an indication, and $14.0(10.0-19.0)$ hours in patients without an indication $(p<0.001)$ (Table 2 and Figure 2). 
Table 1. Baseline Characteristics.

\begin{tabular}{|c|c|c|c|c|c|c|}
\hline & \multicolumn{3}{|c|}{$\begin{array}{l}\text { Without an Indication for } \\
\text { Prone Positioning }\end{array}$} & \multicolumn{3}{|c|}{$\begin{array}{l}\text { With an Indication for } \\
\text { Prone Positioning }\end{array}$} \\
\hline & $\begin{array}{l}\text { No Prone } \\
\text { Positioning } \\
(N=232)\end{array}$ & $\begin{array}{c}\text { Prone } \\
\text { Positioning } \\
(N=217)\end{array}$ & $p$ Value & $\begin{array}{c}\text { No Prone } \\
\text { Positioning } \\
(N=64)\end{array}$ & $\begin{array}{c}\text { Prone } \\
\text { Positioning } \\
(N=221)\end{array}$ & $p$ Value \\
\hline Age, years (median, IQR) & $64.2(11.4)$ & $65.0(10.3)$ & 0.430 & $66.6(9.1)$ & $62.6(11.2)$ & 0.008 \\
\hline Male gender, $N(\%)$ & $171(73.7)$ & $164(73.2)$ & 0.916 & 46 (71.9) & $154(69.1)$ & 0.758 \\
\hline BMI, kg/m² (median, IQR) & $28.5(7.8)$ & $28.1(4.0)$ & 0.490 & $29.2(6.3)$ & $29.3(5.3)$ & 0.841 \\
\hline Chest CT performed, $N(\%)$ & $93(40.1)$ & $82(36.6)$ & 0.500 & $17(26.6)$ & $92(41.3)$ & 0.040 \\
\hline Affected lung parenchyma (\%) & $5(5.3)$ & $2(2.4)$ & 0.212 & $0(0.0)$ & $2(2.2)$ & 0.967 \\
\hline$\leq 25 \%$ & $28(29.8)$ & $28(34.1)$ & & $6(35.3)$ & $27(29.0)$ & \\
\hline $50 \%$ & $34(36.2)$ & $19(23.2)$ & & $5(29.4)$ & $30(32.3)$ & \\
\hline $75 \%$ & $21(22.3)$ & $28(34.1)$ & & $5(29.4)$ & $29(31.2)$ & \\
\hline $100 \%$ & $6(6.4)$ & $5(6.1)$ & & $1(5.9)$ & $5(5.4)$ & \\
\hline Chest X-ray performed, $N(\%)$ & $127(91.4)$ & $135(93.8)$ & 0.501 & $44(93.6)$ & $113(85.6)$ & 0.199 \\
\hline Number of quadrants affected (\%) & & & 0.760 & & & 0.790 \\
\hline 1 & $10(7.9)$ & $10(7.4)$ & & $2(4.5)$ & $8(7.0)$ & \\
\hline 2 & $27(21.4)$ & $36(26.7)$ & & $9(20.5)$ & $27(23.7)$ & \\
\hline 3 & $31(24.6)$ & $34(25.2)$ & & $16(36.4)$ & $32(28.1)$ & \\
\hline 4 & $58(46.0)$ & $55(40.7)$ & & $17(38.6)$ & $47(41.2)$ & \\
\hline Pneumothorax, $N(\%)$ & $0(0.0)$ & $0(0.0)$ & 1.000 & $0(0.0)$ & $1(16.7)$ & 1.000 \\
\hline \multicolumn{7}{|l|}{ Severity of illness } \\
\hline SAPS II (median, IQR) & $35.7(11.7)$ & $36.9(12.8)$ & 0.562 & $35.9(16.1)$ & $37.1(12.8)$ & 0.726 \\
\hline APACHE II (median, IQR) & $16.6(11.3)$ & $18.9(8.5)$ & 0.230 & $16.1(8.0)$ & $19.6(9.3)$ & 0.295 \\
\hline APACHE IV (median, IQR) & $55.2(20.5)$ & $59.6(22.9)$ & 0.197 & $67.2(25.0)$ & $58.6(20.6)$ & 0.086 \\
\hline SOFA (median, IQR) & $8.1(3.2)$ & $9.1(4.4)$ & 0.066 & $8.6(3.2)$ & $8.1(3.8)$ & 0.512 \\
\hline Severity class ARDS (\%) & & & 0.002 & & & 0.607 \\
\hline Mild & $82(36.3)$ & $51(23.5)$ & & $0(0.0)$ & $2(0.9)$ & \\
\hline Moderate & $142(62.8)$ & $157(72.4)$ & & $49(76.6)$ & $154(69.4)$ & \\
\hline Severe & $2(0.9)$ & $9(4.1)$ & & $15(23.4)$ & $66(29.7)$ & \\
\hline $\mathrm{PaO}_{2} / \mathrm{FiO}_{2} \leq 150 \mathrm{~mm} \mathrm{Hg}, N(\%)$ & $90(38.8)$ & $104(47.9)$ & 0.024 & $56(87.5)$ & $189(85.5)$ & 0.600 \\
\hline \multicolumn{7}{|l|}{ Medical history } \\
\hline None & $57(24.6)$ & $46(20.5)$ & 0.315 & $14(21.9)$ & $60(26.9)$ & 0.517 \\
\hline Hypertension, $N(\%)$ & $75(32.3)$ & $75(33.5)$ & 0.842 & $26(40.6)$ & $73(32.7)$ & 0.296 \\
\hline Heart failure, $N(\%)$ & $13(5.6)$ & $12(5.4)$ & 1.000 & $4(6.2)$ & $7(3.1)$ & 0.271 \\
\hline Diabetes, $N(\%)$ & $43(18.5)$ & $57(25.4)$ & 0.089 & $12(18.8)$ & $52(23.3)$ & 0.499 \\
\hline Chronic kidney disease (\%) & $9(3.9)$ & $9(4.0)$ & 1.000 & $2(3.1)$ & $7(3.1)$ & 1.000 \\
\hline $\begin{array}{l}\text { Baseline creatinine, } \mu \mathrm{mol} / \mathrm{L} \\
\text { (median, IQR) }\end{array}$ & $81.1(37.2)$ & $96.2(79.7)$ & 0.021 & $93.2(48.1)$ & $82.5(39.8)$ & 0.121 \\
\hline Liver cirrhosis, $N(\%)$ & $1(0.4)$ & $0(0.0)$ & 1.000 & $1(1.6)$ & $0(0.0)$ & 0.223 \\
\hline $\mathrm{COPD}, N(\%)$ & $16(6.9)$ & $17(7.6)$ & 0.857 & $5(7.8)$ & $24(10.8)$ & 0.640 \\
\hline $\begin{array}{l}\text { Active hematological } \\
\text { neoplasia, } N(\%)\end{array}$ & $5(2.2)$ & $4(1.8)$ & 1.000 & $2(3.1)$ & $0(0.0)$ & 0.049 \\
\hline Active solid neoplasia, N (\%) & $6(2.6)$ & $9(4.0)$ & 0.440 & $0(0.0)$ & $5(2.2)$ & 0.590 \\
\hline Neuromuscular disease, $N(\%)$ & $1(0.4)$ & $0(0.0)$ & 1.000 & $0(0.0)$ & $5(2.2)$ & 0.590 \\
\hline Immunosuppression, $N(\%)$ & $10(4.3)$ & $5(2.2)$ & 0.295 & $1(1.6)$ & $5(2.2)$ & 1.000 \\
\hline \multicolumn{7}{|l|}{ Home medication } \\
\hline Systemic steroids, N (\%) & $13(5.6)$ & $9(4.0)$ & 0.515 & $3(4.7)$ & $5(2.2)$ & 0.383 \\
\hline Inhalation steroids, $N(\%)$ & $26(11.2)$ & $24(10.7)$ & 0.882 & $7(10.9)$ & $29(13.0)$ & 0.831 \\
\hline ACE inhibitor, $N(\%)$ & $44(19.0)$ & $48(21.4)$ & 0.560 & $7(10.9)$ & $38(17.0)$ & 0.329 \\
\hline $\begin{array}{l}\text { Angiotensin II receptor } \\
\text { blocker, } N(\%)\end{array}$ & $22(9.5)$ & $29(12.9)$ & 0.298 & $9(14.1)$ & $21(9.4)$ & 0.352 \\
\hline Beta blocker, N (\%) & $44(19.0)$ & $43(19.2)$ & 1.000 & $18(28.1)$ & $43(19.3)$ & 0.165 \\
\hline Insulin, $N(\%)$ & $17(7.3)$ & $21(9.4)$ & 0.499 & $1(1.6)$ & $9(4.0)$ & 0.467 \\
\hline Metformin, N (\%) & $32(13.8)$ & $44(19.6)$ & 0.103 & $7(10.9)$ & $36(16.1)$ & 0.426 \\
\hline Statin, $N(\%)$ & $63(27.2)$ & $76(33.9)$ & 0.127 & $18(28.1)$ & $76(34.1)$ & 0.450 \\
\hline Calcium channel blockers, $N(\%)$ & $31(13.4)$ & $49(21.9)$ & 0.019 & $15(23.4)$ & $39(17.5)$ & 0.281 \\
\hline
\end{tabular}

Abbreviations: ACE inhibitor, Angiotensin-converting enzyme inhibitor; APACHE, Acute Physiology and Chronic Health Evaluation; ARDS, Acute respiratory distress syndrome; COPD, Chronic obstructive pulmonary disease; IQR, interquartile range; SAPS, Simplified Acute Physiology Score; SOFA, Sequential Organ Failure Assessment; BMI, body mass index. 
Table 2. Duration of Prone Positioning.

\begin{tabular}{|c|c|c|c|}
\hline & $\begin{array}{l}\text { No Indication for } \\
\text { Prone Positioning } \\
\qquad(N=217)\end{array}$ & $\begin{array}{l}\text { Indication for } \\
\text { Prone Positioning } \\
\quad(N=221)\end{array}$ & $p$ Value \\
\hline \multicolumn{4}{|l|}{ Day 0} \\
\hline$n / N(\%)$ & $110 / 217(51)$ & $130 / 221(59)$ & \\
\hline absolute time in a prone position, hours (median, IQR) & $7.5(3.6-12.0)$ & $10.0(6.0-14.4)$ & 0.008 \\
\hline $\begin{array}{l}\text { relative time in a prone position, } \% \text { of total hours * } \\
\text { Day } 1\end{array}$ & $75 \%(65-75)$ & $100 \%(80-100)$ & $<0.001$ \\
\hline$n / N(\%)$ & $167 / 217(77)$ & $180 / 221(81)$ & \\
\hline absolute time in a prone position, hours (median, IQR) & $15(10.0-20.5)$ & $16(11.9-23.1)$ & 0.016 \\
\hline \multicolumn{4}{|l|}{ Day 2} \\
\hline$n / N(\%)$ & $147 / 217(68)$ & $175 / 221(79)$ & \\
\hline absolute time in a prone position, hours (median, IQR) & $12.5(9.0-18.8)$ & $16(10.5-23.5)$ & $<0.001$ \\
\hline $\begin{array}{l}\text { relative time in a prone position, } \% \text { of total hours * } \\
\text { Day } 3\end{array}$ & $52 \%(38-78)$ & $67 \%(44-98)$ & $<0.001$ \\
\hline$n / N(\%)$ & $143 / 217(66)$ & $152 / 221(66)$ & \\
\hline absolute time in a prone position, hours (median, IQR) & $13.8(10.3-18.0)$ & $16.0(11.0-22.0)$ & 0.039 \\
\hline $\begin{array}{c}\text { relative time in a prone position, } \% \text { of total hours * } \\
\text { Total }\end{array}$ & $58 \%(43-75)$ & $67 \%(46-92)$ & 0.039 \\
\hline $\begin{array}{l}\text { Duration of prone positioning per full calendar day } \\
\text { (median, IQR) }\end{array}$ & $14.0(10.0-19.0)$ & $16.0(11.0-23.0)$ & $<0.001$ \\
\hline
\end{tabular}

* Calendar day 0 could last from 0 to $24 \mathrm{~h}$; in patients with no indication day 0 had 10.0 (5.5-16.1) hours, in patients with an indication day 0 had 10.3 (6.0-18.1) hours.
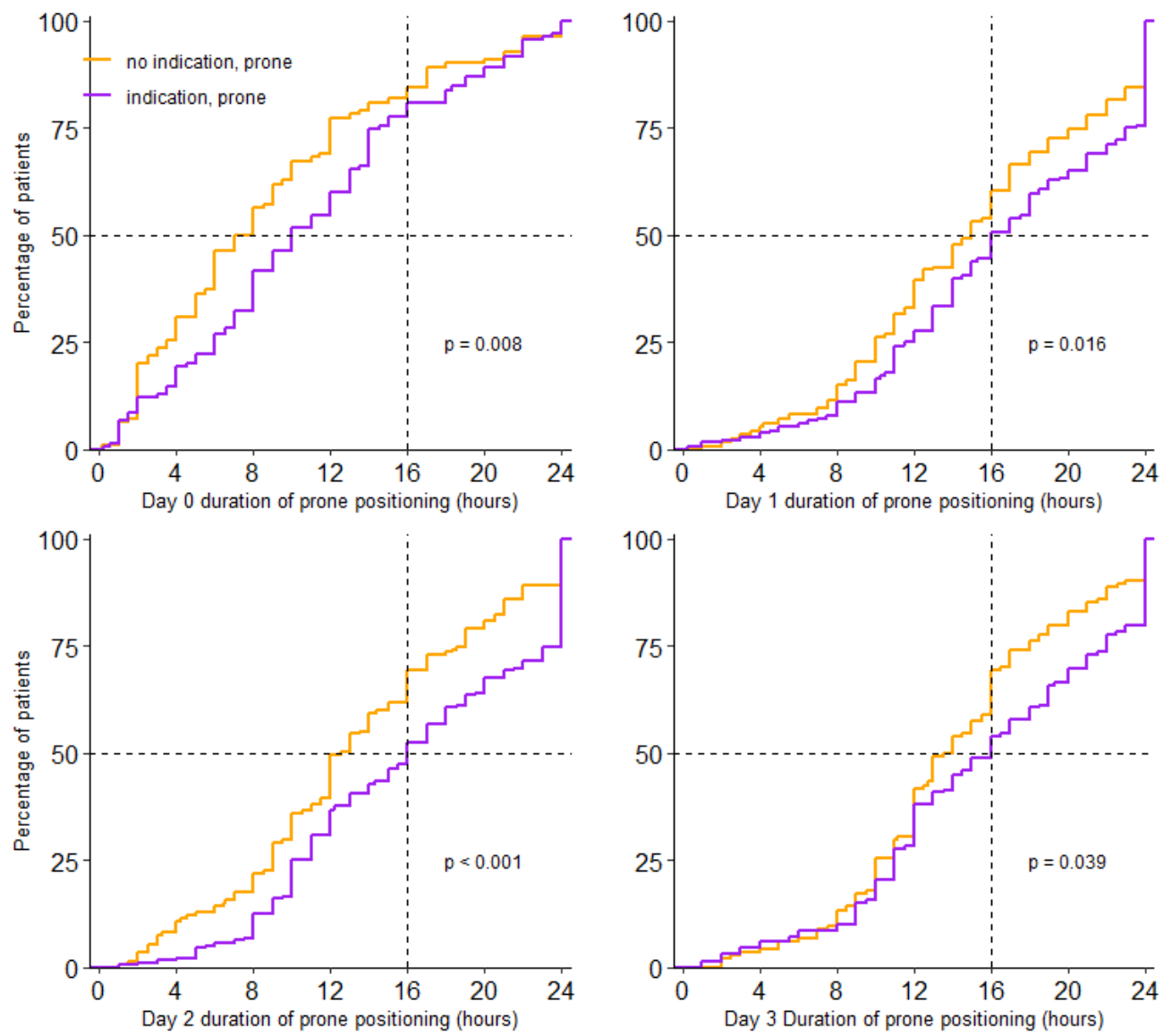

Figure 2. Cumulative distribution duration of prone positioning per day. Duration of prone positioning session for each patient on day 0 to day 3 . 


\subsection{Ventilation Characteristics in the First 4 Calendar Days of Ventilation}

At the start of ventilation, peak airway pressure, driving pressure, compliance, respiratory rate, $\mathrm{FiO}_{2}, \mathrm{PaO}_{2}$, and $\mathrm{SaO}_{2} / \mathrm{FiO}_{2}$ and $\mathrm{PaO}_{2} / \mathrm{FiO}_{2}$, and mechanical power were different between the 4 groups (Table 3 ). In the group without an indication for prone positioning, invasive ventilation differed with regard to the peak and driving pressure. Both were higher in the patients that received prone positioning (Table 3). Driving pressure, compliance, $\mathrm{PaO}_{2} / \mathrm{FiO}_{2}, \mathrm{FiO}_{2}$ and $\mathrm{PaCO}_{2}$ remained different between groups on successive days. Tidal volume was not different between groups on any day of collection of these data. PEEP was only different on day 2 and day $3 ; \mathrm{PaO}_{2}$ was different on day 0 and day 1 (Figure 3, Figure 4 and Figures S1-S8).

Table 3. Ventilatory Characteristics at Start of Ventilation.

\begin{tabular}{|c|c|c|c|c|c|c|}
\hline & \multicolumn{3}{|c|}{$\begin{array}{l}\text { Without an Indication for } \\
\text { Prone Positioning }\end{array}$} & \multicolumn{3}{|c|}{$\begin{array}{l}\text { With an Indication for } \\
\text { Prone Positioning }\end{array}$} \\
\hline & $\begin{array}{l}\text { No Prone } \\
\text { Positioning } \\
(N=232)\end{array}$ & $\begin{array}{c}\text { Prone } \\
\text { Positioning } \\
(N=217)\end{array}$ & $p$ Value & $\begin{array}{l}\text { No Prone } \\
\text { Positioning } \\
(N=64)\end{array}$ & $\begin{array}{c}\text { Prone } \\
\text { Positioning } \\
(N=221)\end{array}$ & $p$ Value \\
\hline Mode of ventilation & 232 & 217 & & 64 & 221 & \\
\hline Volume Control & $23(10.0)$ & $51(22.8)$ & & $6(9.4)$ & $32(14.5)$ & \\
\hline Pressure Control & $127(55.5)$ & $116(51.8)$ & & $33(51.6)$ & $131(59.3)$ & \\
\hline Pressure Support & $7(3.1)$ & $4(1.8)$ & & $0(0.0)$ & $8(3.6)$ & \\
\hline SIMV & $17(7.4)$ & $24(10.7)$ & & $11(17.2)$ & $19(8.6)$ & \\
\hline APRV & $6(2.6)$ & $7(3.1)$ & & $0(0.0)$ & $9(4.1)$ & \\
\hline Intellivent-ASV & $11(4.8)$ & $6(2.7)$ & & $3(4.7)$ & $5(2.3)$ & \\
\hline other & $38(16.6)$ & $16(7.1)$ & & $11(17.2)$ & $17(7.7)$ & \\
\hline Vt, mL/kg PBW (median, IQR) & $6.2(5.7-6.9)$ & $6.2(5.7,6.8)$ & 0.561 & $6.3(5.9-7.1)$ & $6.2(5.7-7.0)$ & 0.295 \\
\hline PEEP (median, IQR) & $12.0(10.0-15.0)$ & $12.0(10.0-15.0)$ & 0.419 & $\begin{array}{c}12.0 \\
(10.0-14.0)\end{array}$ & $\begin{array}{c}12.0 \\
(10.0-14.8)\end{array}$ & 0.848 \\
\hline Ppeak (median, IQR) & $25.5(23.0-29.0)$ & $27.0(24.0-30.0)$ & 0.022 & $\begin{array}{c}27.0 \\
(24.0-30.0)\end{array}$ & $\begin{array}{c}28.0 \\
(25.0-32.0)\end{array}$ & 0.106 \\
\hline Driving pressure (median, IQR) & $13.3(12.0-16.0)$ & $15.0(12.0-16.8)$ & 0.059 & $\begin{array}{c}14.0 \\
(13.0-16.2)\end{array}$ & $\begin{array}{c}15.7 \\
(13.0-18.2)\end{array}$ & 0.030 \\
\hline Mechanical power (median, IQR) & $17.0(13.2-19.8)$ & $16.4(13.6-19.8)$ & 0.861 & $\begin{array}{c}17.7 \\
(13.9-21.4)\end{array}$ & $\begin{array}{c}18.1 \\
(14.8-22.6)\end{array}$ & 0.418 \\
\hline Compliance (median, IQR) & $31.8(26.1-40.6)$ & $29.5(24.1-38.2)$ & 0.120 & $\begin{array}{c}32.3 \\
(26.0-38.2)\end{array}$ & $\begin{array}{c}28.7 \\
(22.3-34.5)\end{array}$ & 0.039 \\
\hline Total respiratory rate (median, IQR) & $20.0(18.0-22.8)$ & $20.0(20.0-24.0)$ & 0.136 & $\begin{array}{c}20.0 \\
(18.0-21.5)\end{array}$ & $\begin{array}{c}21.0 \\
(18.0-25.0)\end{array}$ & 0.014 \\
\hline $\mathrm{FiO}_{2}$ (median, IQR) & $0.6(0.5-0.8)$ & $0.6(0.5-0.8)$ & 0.127 & $0.7(0.6-0.8)$ & $0.8(0.7-1.0)$ & 0.016 \\
\hline $\mathrm{SpO}_{2} / \mathrm{FiO}_{2}$ ratio (median, IQR) & $\begin{array}{c}158.3 \\
(125.5-192.1)\end{array}$ & $\begin{array}{c}153.3 \\
(116.7-180.0)\end{array}$ & 0.022 & $\begin{array}{l}129.0(109.1- \\
141.5)\end{array}$ & $\begin{array}{c}115.1 \\
(96.2-136.5)\end{array}$ & 0.012 \\
\hline End tidal $\mathrm{CO}_{2} \mathrm{mmHg}$ (median, IQR) & $4.8(4.3-5.5)$ & $4.8(4.2-5.5)$ & 0.889 & $4.7(4.3-5.6)$ & $4.9(4.3-5.7)$ & 0.291 \\
\hline NMBA & $89(38.4)$ & $116(51.8)$ & 0.005 & $27(42.2)$ & $133(59.6)$ & 0.015 \\
\hline \multicolumn{7}{|l|}{ Vital signs } \\
\hline $\begin{array}{l}\text { Mean arterial pressure, } \mathrm{mmHg} \\
\text { (median, IQR) }\end{array}$ & $82.5(72.0-98.0)$ & $\begin{array}{c}86.0 \\
(75.0-101.0)\end{array}$ & 0.106 & $\begin{array}{c}87.0 \\
(74.0-97.2)\end{array}$ & $\begin{array}{c}88.5 \\
(75.0-105.2)\end{array}$ & 0.386 \\
\hline $\begin{array}{l}\text { Heart rate, beats per min } \\
\text { (median, IQR) }\end{array}$ & $\begin{array}{c}89.0 \\
(77.0-105.5)\end{array}$ & $\begin{array}{c}91.0 \\
(78.0-105.0)\end{array}$ & 0.380 & $\begin{array}{c}88.5 \\
(76.5-100.2)\end{array}$ & $\begin{array}{c}96.0 \\
(83.0-112.0)\end{array}$ & 0.002 \\
\hline \multicolumn{7}{|l|}{ Laboratory tests } \\
\hline $\mathrm{pH}$ (median, IQR) & $7.4(7.3-7.4)$ & $7.4(7.3-7.4)$ & 0.083 & $7.4(7.3-7.4)$ & $7.3(7.3-7.4)$ & 0.224 \\
\hline $\mathrm{PaO}_{2}$ (median, IQR) & $12.3(10.1-15.6)$ & $11.2(9.5-13.8)$ & 0.001 & $\begin{array}{c}10.4 \\
(9.3-11.8)\end{array}$ & $\begin{array}{c}10.1 \\
(8.9-11.4)\end{array}$ & 0.173 \\
\hline $\mathrm{PaO}_{2} / \mathrm{FiO}_{2}$ ratio (median, IQR) & $\begin{array}{c}161.5 \\
(118.8-212.9)\end{array}$ & $\begin{array}{c}143.8 \\
(106.5-181.3)\end{array}$ & 0.005 & $\begin{array}{c}108.8 \\
(92.2-132.0)\end{array}$ & $\begin{array}{c}96.4 \\
(79.2-119.2)\end{array}$ & 0.005 \\
\hline $\mathrm{PaCO}_{2}$ (median, IQR) & $5.5(4.7-6.1)$ & $5.6(4.9-6.5)$ & 0.037 & $5.9(5.0-6.7)$ & $6.2(5.3-7.3)$ & 0.031 \\
\hline Lactate (median, IQR) & $1.2(0.9-1.5)$ & $1.2(0.9-1.5)$ & 0.713 & $1.2(1.0-1.6)$ & $1.2(0.9-1.5)$ & 0.466 \\
\hline Creatinine, $\mu \mathrm{mol} / \mathrm{L}$ (median, IQR) & $72.0(58.0-91.0)$ & $\begin{array}{c}74.0 \\
(62.0-100.0)\end{array}$ & 0.111 & $\begin{array}{c}80.0 \\
(68.0-96.0)\end{array}$ & $\begin{array}{c}74.0 \\
(57.0-91.2)\end{array}$ & 0.086 \\
\hline
\end{tabular}

Abbreviations: SIMV: Synchronized intermittent mandatory ventilation, ASV: Adaptive Support Ventilation, APRV: Airway pressure release ventilation, PBW: predicted bodyweight, NMBA: neuromuscular blocking agents, Vt, Tidal Volume. 

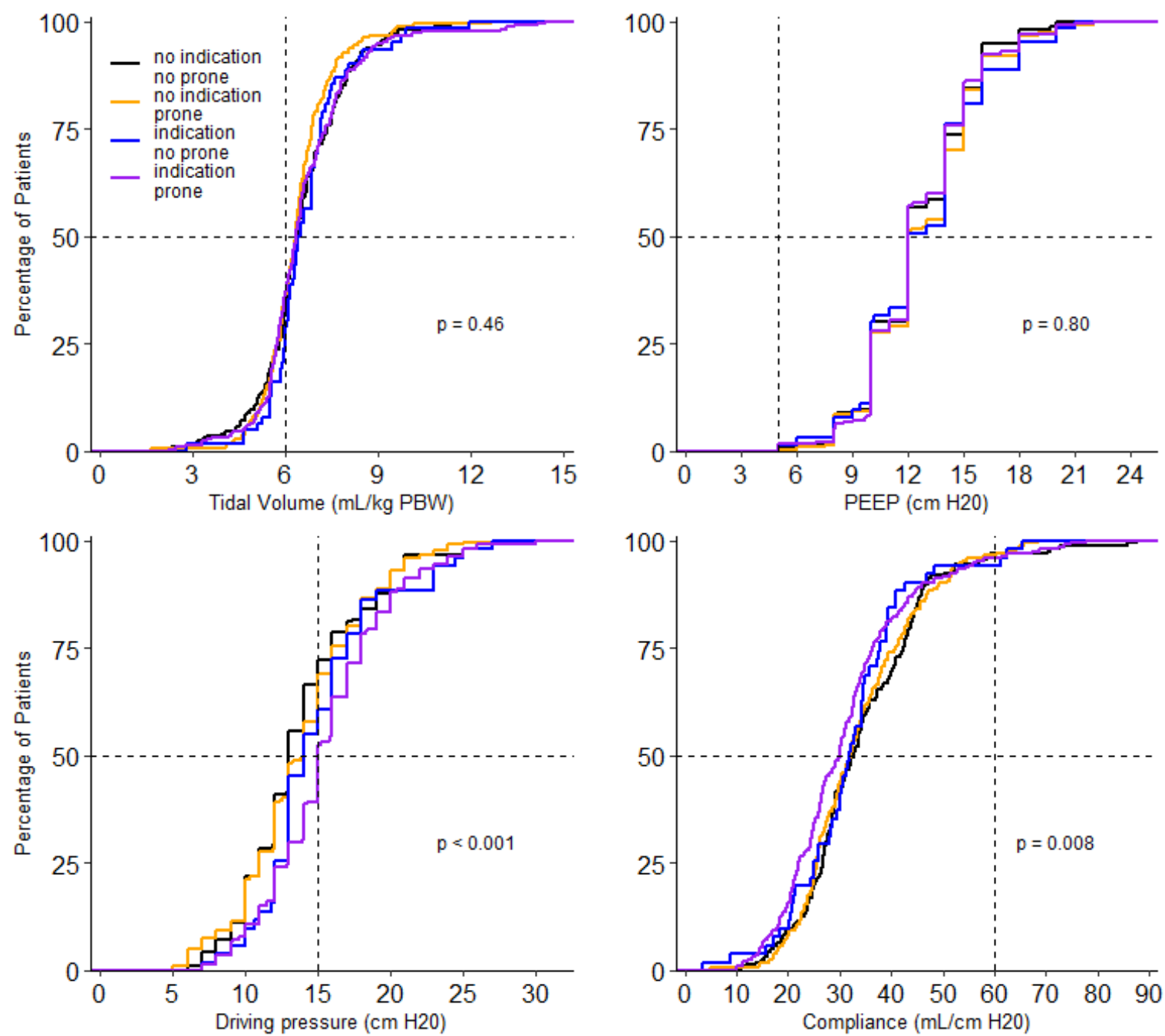

Figure 3. Cumulative distribution of ventilatory parameters on day 0 . Levels of tidal volume, PEEP, driving pressure and compliance for each patient on day 0 .

\subsection{Patient Outcomes}

Mortality at day 28 was lowest in patients with no indication for prone positioning$28.6 \%$ vs. $31.3 \%$ in patients that were not placed in prone position vs. patients that were placed in the prone position. Mortality at day 28 was highest in patients with an indication for prone positioning $-41.3 \%$ vs. $34.1 \%$ in patients that were not placed in prone position vs. patients that were placed in the prone position. Differences between the four groups, though, did not reach statistical significance $(p=0.244)$. Differences in mortality at day 90 between groups followed a similar pattern $(p=0.100)$.

ICU length of stay in patients that survived till ICU discharge was lower in patients that had no indication for prone positioning-a median of 16 (10-25) days vs. 19 (12-33) days, in patients that were not placed in a prone position vs. patients that were placed in a prone position. ICU length of stay in patients that survived till ICU discharge was higher in patients that had an indication for prone positioning-a median of 22 (12-30) days vs. $21(14-34)$ days in patients that were not placed in a prone position vs. patients that were placed in a prone position. 

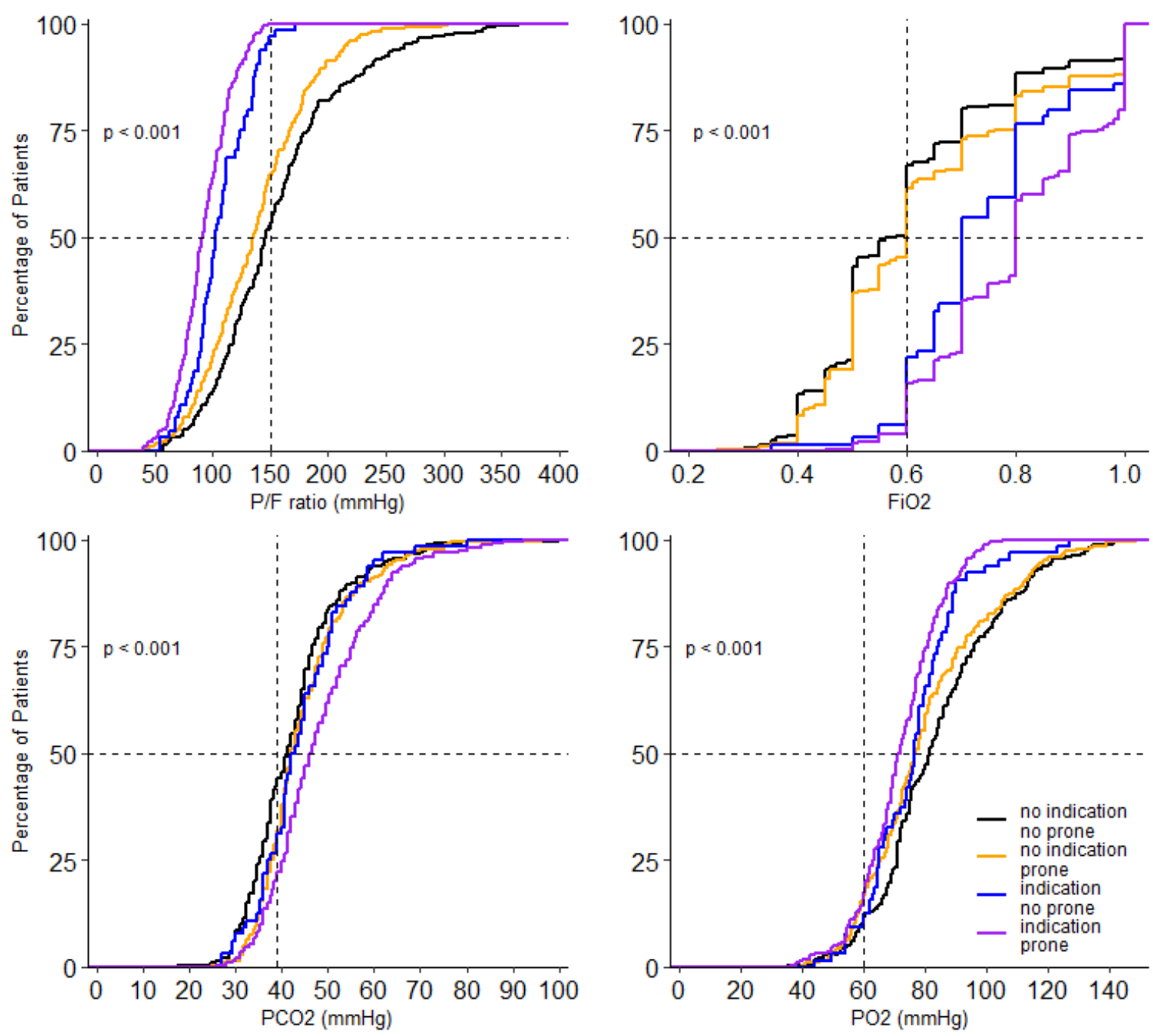

Figure 4. Cumulative distribution of parameters of gas exchange on day 0 . Levels of $\mathrm{P} / \mathrm{F}$ ratio $\mathrm{FiO}_{2}, \mathrm{PO}_{2}$ and $\mathrm{PCO}_{2}$ for each patient on day 0 .

Hospital length of stay in patients that survived till hospital discharge was lower in patients with no indication for prone positioning-a median of 28 (20-40) days vs. 31 (22-) days in patients that were not placed in the prone position vs. patients that were placed in the prone position. Hospital length of stay in patients that survived till hospital discharge was higher in patients with an indication for prone positioning-a median of 31 (21-44) days vs. 35 (24-50) days in patients that were not placed in the prone position vs. patients that were placed in the prone position.

Ventilator free days at day 28 were higher in patients with no indication for prone positioning-a median of $7.0(0.0-17.5)$ days vs. $1.0(0.0-17.00)$ days, in patients that were not placed in the prone position vs. patients that were placed in the prone position. Ventilator free days at day 28 were low in patients with an indication for prone positioninga median of $0.0(0.0-10.0)$ days vs. $0.0(0.0-14.0)$ days, in patients that were not placed in a prone position vs. patients that were placed in a prone position.

Adjusted HRs were different between groups for mortality at day 90, ICU length of stay and hospital length of stay, but not for mortality at day 28 (Figure 5 and Figure S9). 

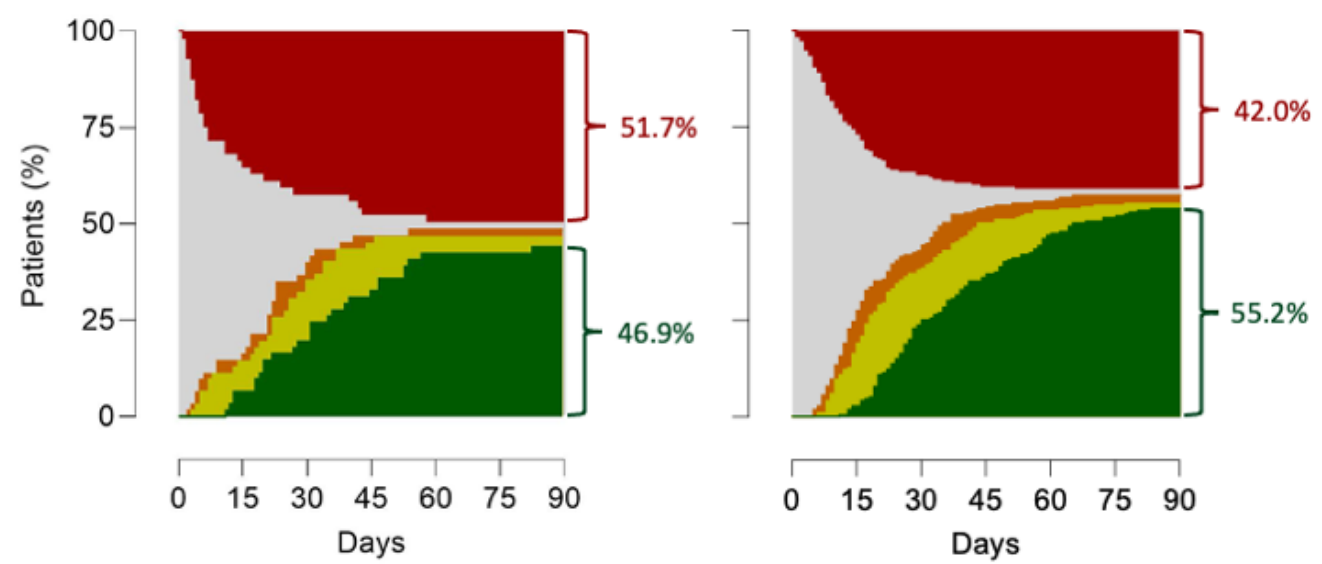

Figure 5. Outcomes. Patient outcomes for the groups of patients with an indication for prone positioning, on the left panel patients are displayed that did not receive prone positioning; on the right panel patients are displayed that did receive prone positioning. HR's for outcomes were (no indication, no prone vs. no indication, prone vs. indication, no prone vs. indication, prone). 28-day mortality: $1.05(0.76-1.45)$ vs. $0.88(0.62-1.26)$ vs. $1.15(0.80-1.54)$ vs. $0.96(0.73-1.26)$ ( $p=0.08)$; 90-day mortality: $0.93(0.67-1.27)$ vs. $0.89(0.64-1.24)$ vs. $1.19(0.88-1.62)$ vs. $0.99(0.76-1.28)$ $(p=0.02)$; ICU discharge: $1.28(1.02-1.61)$ vs. $1.03(0.80-1.33)$ vs. $0.88(0.69-1.12)$ vs. $0.89(0.74-1.08)$ ( $p=0.02)$; Hospital discharge: $1.25(0.99-1.58)$ vs. 1.07 (0.83-1.39) vs. $0.88(0.69-1.13)$ vs. (0.89 $(0.73-1.08)(p=0.01)$; HR's for outcomes in the groups with an indication were (indication, no prone vs. indication, prone); 28-day mortality: 1.30 (0.82-2.07 vs. $0.76(0.48-1.21)(p=0.25)$; 90-day mortality: $1.41(0.93-2.14)$ vs. $0.70(0.46-1.07)(p=0.10)$; ICU discharge: $0.77(0.52-1.14)$ vs. $1.29(0.87-1.91)$ $(p=0.93)$; Hospital discharge: $0.78(0.52-1.18)$ vs. $1.26(0.84-1.90)(p=0.70)$.

\subsection{Factors That Have an Association with Use of Prone Positioning}

ARDS severity and $\mathrm{FiO}_{2}$ were the only factors that were independently associated with the actual use of prone positioning (Table S2).

\subsection{Post Hoc Analysis}

The time-dependent Cox regression analysis did not change the findings (Table S3).

\section{Discussion}

Here, we describe the practice and outcome of prone positioning in patients with ARDS due to COVID-19 that received invasive ventilation in the first 3 months of the national outbreak in the Netherlands. The incidence of prone positioning was high, also in patients not having an indication for this intervention. Sessions of prone positioning were long and lasted longer in patients with an indication. ARDS severity and $\mathrm{FiO}_{2}$ predicted the use of prone positioning.

Our study confirms the high incidence of prone positioning in invasively ventilated COVID-19 patients, as found in other observational studies $[6,9,11,18]$. Studies from before the COVID-19 pandemic showed a remarkable underuse of this intervention in patients with ARDS-in the LUNG SAFE study in 2014, overall use was 7.9\%, and $16.3 \%$ in patients with severe ARDS [19]; in the APRONET study in 2016, overall use was $13.7 \%$, and $32.9 \%$ of patients with severe ARDS [5]. There are several reasons why prone positioning is used more often in COVID-19 patients. It could simply be that the increase of use has continued after LUNG SAFE and APRONET - the implementation of interventions with proven benefits can take many years, also in the ICU setting [20]. It could also be that the poor results of randomized clinical trials that tested alternative ways to improve outcomes, like higher PEEP and recruitment maneuvers, have had a positive effect on the use of prone 
positioning. Last but not least, it could be that COVID-19 ARDS presents as a form of lung injury that may respond better to prone positioning than other forms of ARDS [3]. Indeed, the findings of one randomized clinical trial suggest that prone positioning may be better than higher PEEP in ARDS patients with non-recruitable lung lesions, which may be typical in COVID-19 ARDS, at least at the initiation of invasive ventilation $[3,21]$.

The high incidence of prone positioning was notable in patients not having an indication for this intervention. This may also have been the case in other cohorts, as the reported overall $\mathrm{PaO}_{2} / \mathrm{FiO}_{2}$ ratio in other studies was comparable to that in our cohort $[5,8,14]$, suggesting a similar distribution of ARDS severities and with that a comparable rate of indication for prone positioning. In addition, some of the patients without an indication for prone positioning were actually placed in the prone position. This group had median lower $\mathrm{PaO}_{2} / \mathrm{FiO}_{2}$ ratios, which could be seen as an indication to initiate prone positioning by the clinician. Whether the results of the chest X-rays and lung CT-scans were an indication for the clinician to initiate prone positioning could not be collected. Therefore, we could not comment on or analyze the relationship between imaging and the indication for prone positioning in this cohort.

In our data, there was no difference in mortality at day 28 between groups, but there was a difference in mortality at day 90 . Duration of invasive ventilation is remarkably high in COVID-19 patients, and so is LOS in ICU in these patients [11-30]. This can explain why 28-mortality was not different between the groups, while 90-day mortality was. On the one hand, it could suggest the benefit of this intervention in ARDS due to COVID19 , in line with the findings of the seminal study in patients with ARDS not related to COVID-19 in France [1]. This finding is also in line with the results of one meta-analysis of studies in invasively ventilated COVID-19 patients [23]. On the other hand, it could be that this intervention was foregone in patients with a poor outcome, or in patients with treatment directives. Information on this was not collected in the PRoVENT-COVID study. This explanation, however, seems less likely as there were no differences in any baseline characteristic. The use of NMBA during prone positioning was remarkably lower than in previous studies in the pre-COVID era, in which the incidences were as high as $72 \%$ [23] to $88 \%$ [11]. It should be noted, though, that recommendations regarding the use of NMBA in ARDS patients [24] may have become obsolete after the publication of the more recent ROSE trial [25]. Additionally, recommendations for the use of NMBA, as well as the actual use of NMBA during prone positioning, may depend on local practices, and maybe even on the experiences of the healthcare workers that had to take care of patients in the overwhelming first wave of the COVID-19 pandemic. Notably, a recent study in COVID-19 patients showed NMBA use to be associated with a higher risk of and a longer duration of ventilation and longer ICU LOS, even after propensity matching [26].

A recent study in patients with ARDS due to COVID-19 showed that sustained improvements in oxygenation in response to the first prone positioning session are associated with better outcomes [27]. Recently, the recruitment-to-inflation ratio was suggested as a bedside tool to identify patients that have a high chance of responding well to lung recruitment maneuvers [28]. Unfortunately, we were unable to separate patients based on these approaches, due to the way data were collected.

Prone positioning could come with procedure-related adverse effects $[5,29]$. We did not collect these data. It could be hypothesized that the incidence of adverse events during a pandemic is high due to the stressful and demanding situation, with increased workloads and the lack of experienced staff. However, when dedicated prone position teams are present, as was often the case in the centers in the Netherlands early in the pandemic, the rate of procedure-related adverse events could also be low [30].

The only two factors that had an association with the actual use of prone positioning were ARDS severity and $\mathrm{FiO}_{2}$. This is in line with an earlier observation from before the COVID-19 pandemic [5]. In that study, the major reason for not placing a patient in the prone position was that clinicians deemed hypoxemia not being severe enough. In the current analysis, a $\mathrm{PaO}_{2} / \mathrm{FiO}_{2}$ ratio $<150 \mathrm{mmHg}$ at two successive observations was 
used as a cutoff for the indication for prone positioning. This is more strict than in the previous study.

This study has strengths. First, the data were collected in a short time frame during which general care for COVID-19 patients did not change. Second, the study was designed to minimize bias by strictly adhering to a predefined statistical analysis plan. Third, the study involved one-third of all COVID-19 ARDS patients receiving invasive ventilation in the first months of the national outbreak in the Netherlands, and patients were enrolled in 22 ICUs from university-affiliated hospitals, teaching hospitals and non-teaching hospitals, contributing to its generalizability.

PRoVENT-COVID also has limitations. As in any observational study, the knowledge that care data were being captured could have interfered with practice-for instance, doctors and nurses in participating centers could have been keener to use prone positioning. In line with the study design, the use of late prone positioning, i.e., after the first 4 days of invasive ventilation, was not collected. This means that we could not report on the associations of late prone positioning, if that happened, with outcomes. Additionally, it should be realized that reasons to exclude patients from prone positioning, such as recent tracheal surgery or sternotomy, pregnancy or presence of wounds or burns, were not collected. It is conceivable, though, that these contra-indications were barely present in this cohort. Nevertheless, this may have introduced misclassification bias. The selection of ICUs was based on the personal contacts between steering committee members and ICUs that participated in recent research projects of ventilation, which could have resulted in an overrepresentation of units with more experience in prone positioning, and therefore a higher incidence. Similar to other epidemiological studies, access to patients' data was restricted to data collectors who were granted access only to patients that were labeled eligible for participation by the local doctors-thus, we could not control whether all COVID-19 patients receiving invasive ventilation in participating ICUs were enrolled. Lastly, the national character of PRoVENT-COVID may make these results not representative for other countries.

The findings of this study extend our understanding of the incidence and practice of prone positioning in patients with ARDS due to COVID-19, and the association of this intervention with outcomes. Our findings may have important suggestions for clinical management.

\section{Conclusions}

In this national cohort of patients with ARDS due to COVID-19, prone positioning was frequently used, even in patients that did not have an indication for this intervention. Prone positioning may improve the outcome of invasively ventilated patients with an indication for this intervention. Factors that had an association with its use were ARDS severity and set $\mathrm{FiO}_{2}$.

Supplementary Materials: The following are available online at https://www.mdpi.com/article/ 10.3390/jcm10204783/s1, Table S1: Univariable and Multivariable Model of Covariates Selected for Inclusion in the Final Model Mortality, Table S2: Univariable and Multivariable Model linear mixed model initiation prone positioning, Table S3: Time dependent cox regression analysis, Figure S1: Distribution curves 4 groups tidal volume, PEEP, driving pressure and compliance day 1, Figure S2: Distribution curves 4 groups P/F ratio, FiO2, PO2, PCO2 day 1, Figure S3: Distribution curves 4 groups tidal volume, PEEP, driving pressure and compliance day 2, Figure S4: Distribution curves 4 groups $\mathrm{P} / \mathrm{F}$ ratio, $\mathrm{FiO} 2, \mathrm{PO} 2, \mathrm{PCO} 2$ day 2, Figure S5: Distribution curves 4 groups tidal volume, PEEP, driving pressure and compliance day 3, Figure S6: Distribution curves 4 groups $\mathrm{P} / \mathrm{F}$ ratio, $\mathrm{FiO} 2, \mathrm{PO} 2, \mathrm{PCO} 2$ day 3, Figure S7: Line graphs tidal volume, driving pressure, PEEP and compliance day 0, 1, 2, 3, Figure S8: Line graphs P/F ratio, PO2, PCO2, FiO2 for day 0, 1, 2, 3, Figure S9: Outcomes. 
Author Contributions: Conceptualization, all authors; methodology, all authors; formal analysis, W.S., D.M.P.v.M. and A.S.N.; writing—original draft preparation, W.S., D.M.P.v.M. and C.M.A.V.; writing-review and editing, M.J.S. and F.P.; visualization, W.S., D.M.P.v.M. and A.S.N.; supervision, M.J.S., F.P. and A.S.N.; funding acquisition, W.S., M.J.S. and F.P. All authors have read and agreed to the published version of the manuscript.

Funding: The PRoVENT-COVID study was funded by Amsterdam UMC, location AMC, Amsterdam, The Netherlands. Research time from W. Stilma was funded by a personal (PhD fellowship) grant from NWO Netherlands Organisation for Scientific Research, number 023.011.016.

Institutional Review Board Statement: The study protocol was approved by the ethics committee in Amsterdam UMC, Amsterdam, the Netherlands (registration number W20_157 \# 20.171); the need for in-dividual patient informed consent was waived due to the observational nature of the study.

Informed Consent Statement: Patient consent was waived due to the observational nature of the study.

Data Availability Statement: Access to source data will be made available through local, regional and national anonymized datasets on request, and after agreement of the PRoVENT-COVID steering committee.

Conflicts of Interest: The authors declare no conflict of interest.

\section{Appendix A}

PRoVENT-COVID Collaborative Group:

S. Ahuja; J.P. van Akkeren; A.G. Algera; C.K. Algoe; R.B. van Amstel; A. Artigas; O.L. Baur; P. van de Berg; A.E. van den Berg; D.C.J.J. Bergmans; D.I. van den Bersselaar; F.A. Bertens; A.J.G.H. Bindels; M.M. de Boer; S. den Boer; L.S. Boers; M. Bogerd; L.D.J. Bos; M. Botta; J.S. Breel; H. de Bruin; S. de Bruin; C.L. Bruna; L.A. Buiteman-Kruizinga; O.L. Cremer; R.M. Determann; W. Dieperink; D.A. Dongelmans; H.S. Franke; M.S. GalekAldridge; M.J. de Graaff; L.A. Hagens; J.J. Haringman; S.T. van der Heide; P.L.J. van der Heiden; N.F.L. Heijnen; S.J.P. Hiel; L.L. Hoeijmakers; L. Hol; M. W. Hollmann; M.E. Hoogendoorn; J. Horn; R. van der Horst; E.L.K. Ie; D. Ivanov; N.P. Juffermans; E. Kho; E.S. de Klerk; A.W.M.M. Koopman-van Gemert; M. Koopmans; S. Kucukcelebi; M.A. Kuiper; D.W. de Lange; N. van Mourik; L. Morales-Quinteros; S.G. Nijbroek; M. Onrust; E.A.N. Oostdijk; F. Paulus; C.J. Pennartz; J. Pillay; L. Pisani; I.M. Purmer; T.C.D. Rettig; J.P. Roozeman; M.T.U. Schuijt; M.J. Schultz; A. Serpa Neto; M.E. Sleeswijk; M.R. Smit; P.E. Spronk; W. Stilma; A.C. Strang; A. M. Tsonas; P.R. Tuinman; C.M.A. Valk; F.L. Veen-Schra; L.I. Veldhuis; P. van Velzen; W.H. van der Ven; A.P.J. Vlaar; P. van Vliet; P.H.J. van der Voort; L. van Welie; H.J.F.T. Wesselink; H.H. van der Wier-Lubbers; B. van Wijk; T. Winters; W.Y. Wong; A.R.H. van Zanten.

\section{References}

1. Guérin, C.; Reignier, J.; Richard, J.-C.; Beuret, P.; Gacouin, A.; Boulain, T.; Mercier, E.; Badet, M.; Mercat, A.; Baudin, O.; et al. Prone Positioning in Severe Acute Respiratory Distress Syndrome. N. Engl. J. Med. 2013, 368, 2159-2168. [CrossRef]

2. Sud, S.; Friedrich, J.; Adhikari, N.K.J.; Taccone, P.; Mancebo, J.; Polli, F.; Latini, R.; Pesenti, A.; Curley, M.; Fernandez, R.; et al. Effect of prone positioning during mechanical ventilation on mortality among patients with acute respiratory distress syndrome: A systematic review and meta-analysis. Can. Med. Assoc. J. 2014, 186, E381-E390. [CrossRef] [PubMed]

3. Constantin, J.-M.; Jabaudon, M.; Lefrant, J.-Y.; Jaber, S.; Quenot, J.-P.; Langeron, O.; Ferrandière, M.; Grelon, F.; Seguin, P.; Ichai, C.; et al. Personalised mechanical ventilation tailored to lung morphology versus low positive end-expiratory pressure for patients with acute respiratory distress syndrome in France (the LIVE study): A multicentre, single-blind, randomised controlled trial. Lancet Respir. Med. 2019, 7, 870-880. [CrossRef]

4. Duggal, A.; Rezoagli, E.; Pham, T.; McNicholas, B.A.; Fan, E.; Bellani, G.; Rubenfeld, G.; Pesenti, A.M.; Laffey, J.G.; LUNG SAFE Investigators; et al. Patterns of Use of Adjunctive Therapies in Patients with Early Moderate to Severe ARDS: Insights from the LUNG SAFE Study. Chest 2020, 157, 1497-1505. [CrossRef] [PubMed]

5. Guérin, C.; Beuret, P.; Constantin, J.M.; Bellani, G.; Garcia-Olivares, P.; Roca, O.; Meertens, J.H.; Maia, P.A.; Becher, T.; Peterson, J.; et al. A prospective international observational prevalence study on prone positioning of ARDS patients: The APRONET (ARDS Prone Position Network) study. Intensive Care Med. 2018, 44, 22-37. [CrossRef] [PubMed] 
6. Ferrando, C.; Suarez-Sipmann, F.; Mellado-Artigas, R.; Hernández, M.; Gea, A.; Arruti, E.; Aldecoa, C.; Martínez-Pallí, G.; Martínez-González, M.A.; Slutsky, A.S.; et al. Correction to: Clinical features, ventilatory management, and outcome of ARDS caused by COVID-19 are similar to other causes of ARDS. Intensive Care Med. 2021, 47, 144-146. [CrossRef]

7. Schultz, M.J. High versus low PEEP in non-recruitable collapsed lung tissue: Possible implications for patients with COVID-19. Lancet Respir. Med. 2020, 8, e44. [CrossRef]

8. Grasselli, G.; Cattaneo, E.; Florio, G.; Ippolito, M.; Zanella, A.; Cortegiani, A.; Huang, J.; Pesenti, A.; Einav, S. Mechanical ventilation parameters in critically ill COVID-19 patients: A scoping review. Crit. Care 2021, 25, 115. [CrossRef]

9. Cummings, M.J.; Baldwin, M.R.; Abrams, D.; Jacobson, S.D.; Meyer, B.J.; Balough, E.M.; Aaron, J.G.; Claassen, J.; Rabbani, L.E.; Hastie, J.; et al. Epidemiology, clinical course, and outcomes of critically ill adults with COVID-19 in New York City: A prospective cohort study. medRxiv 2020. [CrossRef]

10. Grasselli, G.; Tonetti, T.; Protti, A.; Langer, T.; Girardis, M.; Bellani, G.; Laffey, J.; Carrafiello, G.; Carsana, L.; Rizzuto, C.; et al. Pathophysiology of COVID-19-associated acute respiratory distress syndrome: A multicentre prospective observational study. Lancet Respir. Med. 2020, 8, 1201-1208. [CrossRef]

11. COVID-ICU Group on Behalf of the REVA Network and the COVID-ICU Investigators. Clinical characteristics and day-90 outcomes of 4244 critically ill adults with COVID-19: A prospective cohort study. Intensive Care Med. 2021, 47, 60-73. [CrossRef]

12. Boers, N.S.; Botta, M.; Tsonas, A.M.; Algera, A.G.; Pillay, J.; Dongelmans, D.A.; Horn, J.; Vlaar, A.P.J.; Hollmann, M.W.; Bos, L.D.J.; et al. PRactice of VENTilation in Patients with Novel Coronavirus Disease (PRoVENT-COVID): Rationale and protocol for a national multicenter observational study in The Netherlands. Ann. Transl. Med. 2020, 8, 1251. [CrossRef]

13. Botta, M.; Tsonas, A.M.; Pillay, J.; Boers, L.S.; Algera, A.G.; Bos, L.D.J.; Dongelmans, D.A.; Hollmann, M.W.; Horn, J.; Vlaar, A.P.J.; et al. Ventilation management and clinical outcomes in invasively ventilated patients with COVID-19 (PRoVENT-COVID): A national, multicentre, observational cohort study. Lancet. Respir. Med. 2021, 9, 139-148. [CrossRef]

14. PRactice of VENTilation in Patients with Coronavirus Disease 2019 (PRoVENT-COVID)—Rationale, Study Protocol and Statistical Analysis Plans for a National Multicenter Observational Study in The Netherlands. Available online: https://sites.google.com/ view/provent-covid/provent-covid (accessed on 19 April 2021).

15. Ferguson, N.D.; Fan, E.; Camporota, L.; Antonelli, M.; Anzueto, A.; Beale, R.; Brochard, L.; Brower, R.; Esteban, A.; Gattinoni, L.; et al. The Berlin definition of ARDS: An expanded rationale, justification, and supplementary material. Intensive Care Med. 2012, 38, 1573-1582. [CrossRef] [PubMed]

16. Force, A.D.T.; Ranieri, V.M.; Rubenfeld, G.D.; Thompson, B.T.; Ferguson, N.D.; Caldwell, E.; Fan, E.; Camporota, L.; Slutsky, A.S. Acute respiratory distress syndrome: The Berlin Definition. JAMA 2012, 307, 2526-2533. [CrossRef]

17. R Foundation for Statistical Computing. R: A Language and Environment for Statistical Computing; R Foundation for Statistical Computing: Vienna, Austria, 2018.

18. Mathews, K.S.; Soh, H.; Shaefi, S.; Wang, W.; Bose, S.; Coca, S.; Gupta, S.; Hayek, S.S.; Srivastava, A.; Brenner, S.K.; et al. Prone Positioning and Survival in Mechanically Ventilated Patients with Coronavirus Disease 2019-Related Respiratory Failure. Crit. Care Med. 2021, 49, 1026-1037. [CrossRef] [PubMed]

19. Bellani, G.; Laffey, J.G.; Pham, T.; Fan, E.; Brochard, L.; Esteban, A.; Gattinoni, L.; Van Haren, F.; Larsson, A.; McAuley, D.F.; et al. Epidemiology, Patterns of Care, and Mortality for Patients with Acute Respiratory Distress Syndrome in Intensive Care Units in 50 Countries. JAMA 2016, 315, 788-800. [CrossRef] [PubMed]

20. Morris, Z.; Wooding, S.; Grant, J. The answer is 17 years, what is the question: Understanding time lags in translational research. J. R. Soc. Med. 2011, 104, 510-520. [CrossRef] [PubMed]

21. Schultz, M.J.; Neto, A.S.; Paulus, F. Battling COVID-19-related mortality: From a fight for ventilators to a cry for oxygen. Lancet Respir. Med. 2021, 9, 939-941. [CrossRef]

22. Ferrando, C.; Mellado-Artigas, R.; Gea, A.; Arruti, E.; Aldecoa, C.; Bordell, A.; Adalia, R.; Zattera, L.; Ramasco, F.; Monedero, P.; et al. Patient characteristics, clinical course and factors associated to ICU mortality in critically ill patients infected with SARS-CoV-2 in Spain: A prospective, cohort, multicentre study. Rev. Esp. Anestesiol. Reanim. 2020, 67, 425-437. [CrossRef]

23. Chua, E.X.; Zahir, S.M.I.S.M.; Ng, K.T.; Teoh, W.Y.; Bin Hasan, M.S.; Ruslan, S.R.B.; Abosamak, M.F. Effect of prone versus supine position in COVID-19 patients: A systematic review and meta-analysis. J. Clin. Anesth. 2021, 74, 110406. [CrossRef]

24. Murray, M.J.; DeBlock, H.F.; Erstad, B.L.; Gray, A.W.; Jacobi, J.; Jordan, C.J.; McGee, W.T.; McManus, C.; Meade, M.O.; Nix, S.A.; et al. Clinical practice guidelines for sustained neuromuscular blockade in the adult critically ill patient: 2016 updateexecutive summary. Am. J. Health Pharm. 2017, 74, 76-78. [CrossRef] [PubMed]

25. National Heart, Lung, and Blood Institute PETAL Clinical Trials Network; Moss, M.; Huang, D.T.; Brower, R.G.; Ferguson, N.D.; Ginde, A.A.; Gong, M.N.; Grissom, C.K.; Gundel, S.; Hayden, D.; et al. Early Neuromuscular Blockade in the Acute Respiratory Distress Syndrome. N. Engl. J. Med. 2019, 380, 1997-2008. [CrossRef] [PubMed]

26. Bassi, G.L.; Gibbons, K.; Suen, J.; Dalton, H.; White, N.; Barnett, A.; Fraser, J. COVID-19 Critical Care Consortium Neuromuscular Blocking Agents in Critically-Ill COVID-19 Patients Requiring Mechanical Ventilation. In TP48. TP048 COVID: ARDS CLINICAL STUDIES; American Thoracic Society: New York, NY, USA, 2021; p. A2489.

27. Scaramuzzo, G.; Gamberini, L.; Tonetti, T.; Zani, G.; Ottaviani, I.; Mazzoli, C.A.; Capozzi, C.; Giampalma, E.; Reggiani, M.L.B.; Bertellini, E.; et al. Sustained oxygenation improvement after first prone positioning is associated with liberation from mechanical ventilation and mortality in critically ill COVID-19 patients: A cohort study. Ann. Intensive Care 2021, 11, 63. [CrossRef] [PubMed] 
28. Cour, M.; Bussy, D.; Stevic, N.; Argaud, L.; Guérin, C. Differential effects of prone position in COVID-19-related ARDS in low and high recruiters. Intensive Care Med. 2021, 47, 1044-1046. [CrossRef]

29. Kallet, R.H. A Comprehensive Review of Prone Position in ARDS. Respir. Care 2015, 60, 1660-1687. [CrossRef] [PubMed]

30. Rodriguez-Huerta, M.D.; Diez-Fernandez, A.; Rodriguez-Alonso, M.J.; Robles-Gonzalez, M.; Martin-Rodriguez, M.; GonzalezGarcia, A. Nursing care and prevalence of adverse events in prone position: Characteristics of mechanically ventilated patients with severe SARS-CoV-2 pulmonary infection. Nurs. Crit. Care 2021. [CrossRef] 